\title{
Exemestane/Ribociclib Regimen
}

National Cancer Institute

\section{Source}

National Cancer Institute. Exemestane/Ribociclib Regimen. NCI Thesaurus. Code

C156371.

A chemotherapy regimen consisting of exemestane and ribociclib that may be used in the treatment of hormone receptor (HR)-positive advanced or metastatic breast cancer. 